\title{
Correction to: Risk reduction of long-term major adverse cardiovascular events after endoscopic thoracic sympathectomy in palmar hyperhidrosis
}

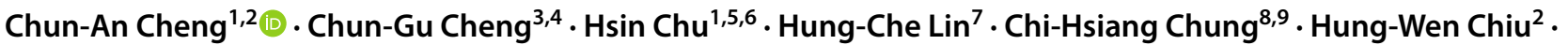 \\ Wu-Chien Chien ${ }^{8,9}$
}

Published online: 2 June 2018

(c) Springer-Verlag GmbH Germany, part of Springer Nature 2018

\section{Correction to: Clin Auton Res (2017) 27:393-400 https://doi.org/10.1007/s10286-017-0464-0}

There is a typographical error in the International Classification of Disease Ninth Revision, Clinical Modification (ICD9-CM) diagnostic code used for palmar hyperhidrosis. The published manuscript wrongly reports that the ICD-9-CM code used for palmar hyperhidrosis was 708.8 (which indicates a diagnosis of "Other specified urticaria"), when, in actuality, the correct code 780.8 ("Hyperhidrosis") was applied. The authors regret this typographical error.
The original article can be found online at https://doi.org/10.1007/ s10286-017-0464-0.

\section{Hung-Wen Chiu}

hwchiu@tmu.edu.tw

$\triangle$ Wu-Chien Chien

chienwu@ndmctsgh.edu.tw

1 Department of Neurology, Tri-Service General Hospital, National Defense Medical Center, Taipei, Taiwan

2 Graduate Institute of Biomedical Informatics, Taipei Medical University, No. 250, Wu-Hsing Street, 110, Taipei, Taiwan

3 Department of Emergency, Armed Taoyuan General Hospital, Taoyuan, Taiwan

4 Department of Emergency and Critical Medicine, Wan Fang Hospital, Taipei Medical University, Taipei, Taiwan

5 Graduate Institute of Aerospace and Undersea Medicine, National Defense Medical Center, Taipei, Taiwan
6 Aviation Physiology Research Laboratory, Gangshan Branch of Kaohsiung Armed Forces General Hospital, Kaohsiung, Taiwan

7 Department of Otolaryngology-Head and Neck Surgery, Tri-Service General Hospital, National Defense Medical Center, Taipei, Taiwan

8 Department of Medical Research, Tri-Service General Hospital, National Defense Medical Center, 7115R, No.325, Section 2, Cheng-Kung Road, Neihu District, Taipei 11490, Taiwan, ROC

9 School of Public Health, National Defense Medical Center, Taipei, Taiwan 